\title{
ON THE OPTIMAL CONTROL OF VARIATIONAL INQQUALITIES
}

\section{F. Patrone}

Istituto Matematico, Universita di Genova via I. B. Alberti 4, I6I32 GENOVA (ITAJY)

I. An existence theorem.

We are given a real and reflexive Banach space $V$, with dual $V '$, an operator $A: V \rightarrow V$ and e function $j: V \rightarrow(-\infty,+\infty]$.

We consider variational inequalities of the following type: find $y \in V$ such that:

$$
\text { (I.I) } \quad\langle A y, z-y\rangle+j(z)-j(y) \geq\langle\delta, z-y\rangle \quad \forall z \in V \text {, }
$$

where $g \in V^{\prime}$ and $\langle\cdot, \cdot\rangle$ is the pairing between $V^{\prime}$ and $V$.

We assume A a peudo-monotone operator, i.e:

$$
\left\{\begin{array}{l}
\text { a) A is bounded (on bounded subsets) } \\
\text { b) } \left.y_{n} \rightarrow y \text { and } \frac{I_{n \rightarrow \infty}}{n \rightarrow y_{n}}, y_{n}-y\right\rangle \leq 0 \text { imply } \\
\frac{I i_{n}}{n \rightarrow \infty}\left\langle A_{n}, y_{n}-z\right\rangle \geq\langle A y, y-z\rangle \quad \forall z \in V .
\end{array}\right.
$$

$j$ is a convex, proper $(i, e . j \not \neq+\infty)$ and lower semicontinuous func tion.

It is well known that inequalities of the type (I.I), for any given $g \in V^{\prime}$, have a solution (see J.I.Iions [4]), if the following coercivity hypothesis is setisfied:

$$
(I .3)\left\{\begin{array}{l}
\exists z_{0} \in V \text { such that } j\left(z_{0}\right)<+\infty \text { and } \\
\frac{\left\langle A z, z-z_{0}\right\rangle+j(z)}{\|z\|} \rightarrow+\infty \text { when }\|z\| \rightarrow \infty .
\end{array}\right.
$$

We shall deal with the following control problem. The space of controls is U, a real and reflexive Banech space; the set of admissi ble controls is $U_{a d}$, a closed convex (non empty) subset of $U ; B: J \longrightarrow V$ ' is a map such that $u_{n} \rightarrow \vec{U} u$ implies $B u_{n} \underset{V}{\longrightarrow} B u$.

For any given $u \in \mathrm{U}_{a d}$, the state $y=y(u)$ is given by the solution 
(not necessarily unique) of the following inequallty ( $f \in V^{\prime}$ is fixed): (I.4) $\quad\langle\mathrm{Ay}, \mathrm{z}-\mathrm{y}\rangle+\mathrm{j}(\mathrm{z})-\mathrm{j}(\mathrm{y}) \geq\langle\mathrm{f}+\mathrm{Bu}, \mathrm{z}-\mathrm{y}\rangle \quad \forall \mathrm{z} \in \mathrm{V}$.

The cost is assumed to be quadratic: $J(u, y(u))=\left\|_{y}(u)-z a\right\|_{V}^{2}+\nu\left\|_{u}\right\|_{U}^{2}, \nu \geq 0$ (a linear and continuous obser vation operator might as well be considered).

The control problem is: minimize $J$ on $\mathrm{Ud}$.

We have the following theorem:

Theorem I.I Under the hypotheses above, if we have:

(I.5) either $\nu>0$ or $U_{\text {ad }}$ is bounded,

there exists an optimal pair $(u, y(u))$.

We give here a brief sketch of the proof. Weftake a minimizing sequen ce $\left\{u_{n}, y_{n}\right\}_{n \in \mathbb{N}}$, where $y_{n}$ is selected among the solutions of $(I .4)$ corresponding to $u_{n}$. It follows from hypothesis (I.5) and the coercivity hypothesis (I.3) that we can extract a subsequence $\left\{u_{n_{i}}, y_{n_{i}}\right\}_{i \in \mathbb{N}}$ such that $u_{n_{i}} \longrightarrow u$ and $y_{n_{i}} \longrightarrow y$ in $\nabla$. The compactness of $B$, the lower semicontinuity of $j$ and the pseudo-monotonicity of $A$ allow us to pass to the limit in the inequality and to prove that $\mathrm{y}$ is a solution corresponding to $u$ of (I.4). To conclude, the weak lower semicontinuity of $J$ on $J \times V$ assure us that $\{u, J\}$ is an optimal control-state peir.

Remark I A particular case of inequality (I.I) is: $(I .6)$
$\langle A y, z-y\rangle \geq\langle g, z-y\rangle$
$\forall \mathrm{z} \in \mathrm{K}$,

where $K$ is a closed convex subset of V.It suffices to define $j(z)=\oint_{K}(z)$ where $\delta_{K}$ is the indicator function of $K$, i.e.:

$\delta_{K}(z)=\left\{\begin{array}{cc}0 & \text { if } z \in K \\ +\infty & \text { if } z \notin K\end{array}\right.$

Remark 2 Generally we have not uniqueness of the optimal control. A very simple counterexample is the following $(V=0=R)$. The inequality is given by $(B u=u+\sqrt{2})$ : 


$$
\text { (I.7) } \quad y \cdot(z-y) \geq(u+\sqrt{2}) \cdot(z-y) \quad \forall z \in[0, I]
$$

The (unique) solution of $(I .7)$ is given by $y(u)=\operatorname{Pr}_{K}(u+\sqrt{2})$, the projection of $u+\sqrt{2}$ on $K=[0, I]$.

If we set $U_{a d}=0$ and $J(u)=u^{2}+(y(u))^{2}$, both $u=0$ and $u=-\frac{\sqrt{2}}{2}$ are optimal controls.

Former results on the optimal control of variational inequalities may be found in J.P.Yron [6], or in J.I.Lions [3] (here a special case is considered); see also R.Kluge [2], and the bibliography Iisted there for further references.

\section{Further results on the control of variational inequalities.}

The existence theorem previously given can be extended to more general cost functionals, provided that the operator A is actually a monotone hemicontinuous and bounded operator. To do this, we need the following theorem of F. B.Browder [I] on the sequential Iower semicontinuity of certain types of functionals.

Theorem 2.I We are given three real Banach spaces $X, X_{1}, X_{2}$; a map $h: x_{1} X_{2} \rightarrow R$ such that:

a) $h\left(x_{1}, x_{2}\right)$ is convex and strongly continuous in $x_{1}$ when $x_{2}$ is $f 1 x \in d$

b) $h\left(x_{1}, x_{2}\right)$ is strongly continuous in $x_{2}$, when $x_{1}$ is fixed, and uniformly continuous in $x_{2}$ when $x_{1}$ varies in bounded subsets. We are given also $I: X \rightarrow X_{1}$, a linear and continuous map, and $\mathrm{M}: \mathrm{X} \rightarrow \mathrm{X}_{2}$ a map sequentially continuous from the weak to the strong topology. Then, setting $J(u)=h(I u, M u): X \rightarrow R, J$ is weakly sequentially Lower semicontinuous. 
We note that in Browder's theorem it is required that $M$ is a linear map, but this hypothesis can be suppressed, as can be easily verified.

If we require that A is a strongly monotone operator, we can apply Browder's theorem to our control problem: to do this we note that under this assumption the map $G: U \rightarrow V$, which associates to the control $u$ the corresponding (unique) state $y(u)$, is sequentially continuous from weak to strong. If we define $X=U, X_{2}=V$ and $M=G$, while $h$, $\mathrm{X}_{1}$ and $\mathrm{L}$ are as in Browder's theorem, we obtain that the map $J(u)=h(\mathrm{Iu}, \mathrm{Mu})$ is sequentially lower semicontinuous on $U$. This fact gives us an existence theorem if assume that the set of admissible controls $\mathrm{U}_{\mathrm{ad}}$ is bounded.

To conclude, we give here briefly a result on the sensitivity of the control problem. We consider variational inequalities of type (I.6), where A is a strictly monotone hemicontinuous and bounded operator. We perturb $A, K, z_{d}$ and $f$. More precisely, we consider a sequence of problems like this:

$$
\begin{aligned}
\left(P_{n}\right): & \text { minimize } J_{n}(v)=\left\|_{y_{n}}(v)-z_{d_{n}}\right\|_{V}^{2}+\nu\left\|_{v}\right\|_{U}^{2} \\
& \text { for } v \in U_{\text {ad }} \text { and } y_{n}(v) \text { the solution of: } \\
& \left\langle A_{n} y_{n}(v), z-y_{n}(v)\right\rangle \geq\left\langle f_{n}+B v, z-y_{n}(v)\right\rangle \quad \forall z \in K_{n}
\end{aligned}
$$

The initial problem is:

$$
\begin{aligned}
\left(P_{0}\right): & \text { minimize } J_{0}(v)=\left\|_{0}(v)-z_{d_{0}}\right\|_{V}^{2}+\nu \|_{0}^{2} \\
& \text { for } v \in J_{a d} \text { and } J_{0}(v) \text { the solution of: } \\
& \left\langle A_{0} y_{0}(v), z-y_{0}(v)\right\rangle \geq\left\langle f_{0}+B v, z-y_{0}(v)\right\rangle \quad \forall z \in K_{0}
\end{aligned}
$$

If we assume that $f_{n} \longrightarrow f_{0}, z_{d_{n}} \rightarrow z_{d_{0}}$, and that $A_{n}, K_{n}$ and $A_{0}, K_{0}$ verify the hypotheses introduced by U.Mosco in [5] to prove the strong convergence of solutions of perturbed variational inequalities, then we 
obtain the following result:

Theorem 2.2 Given a sequence of optimal controls $u_{n}$ for $P_{n}$, we can extract a subsequence $u_{n_{i}}$ weakly converging to an optimal control $\bar{u}$ for $P_{0}$; the corresponding states $y_{n_{i}}$ converge strongly to $\bar{y}=y_{0}(\bar{u})$.

Let us give here a brief sicetch of the proof. Our aim is to prove the boundedness of $u_{n}$, and this is obvious if we prove that $J_{n}\left(u_{n}\right)$ is such. To prove this fact, we note that $J_{n}\left(u_{n}\right) \leq J_{n}\left(u_{0}\right)$, where $u_{0}$ is an optimal control for $P_{0}$. But $J_{n}\left(u_{0}\right)$ is bounded because the states $\mathrm{y}_{n}^{\prime}=\mathrm{y}_{0}\left(u_{n}\right)$ converge strongly to $\mathrm{y}_{0}$; more precisely we obtain that: (2.I) $\forall \varepsilon>0, \exists n(\varepsilon)$ s.t. $\left|J_{n}\left(u_{0}\right)-J_{0}\left(u_{0}\right)\right|<\varepsilon$. This fact implies the boundedness of $J_{n}\left(u_{n}\right)$, so of $u_{n}$. If we consider a subsequence $u_{n_{1}} \rightarrow \bar{u}$, we have $B u_{n_{i}} \longrightarrow B \bar{u}:$ from this follows that $\mathrm{y}_{\mathbf{n}_{i}}\left(u_{n_{i}}\right) \rightarrow \mathrm{y}_{0}(\bar{u})$. To conclude, the weak lower semicontinuity of the costs $J$ imply that $\frac{\text { limJ }}{n \rightarrow \infty}\left(u_{n}\right) \geq J_{0}(\bar{u})$. From this, and from (2.I), we get that $J_{0}(\bar{u}) \leq J_{0}\left(u_{0}\right)+\varepsilon$ for every $\varepsilon>0$, so $J_{0}(\bar{u}) \leq J_{0}\left(u_{0}\right)$. But $J_{0}\left(u_{0}\right) \leq J_{0}(\vec{u})$ for definition of $u_{0}$ : this means that $\bar{u}$ is actually an optimal control for ${ }_{0}$. 


\section{References}

1. BROWDER,F.E. "Remarks on the Direct Method of the Calculus of Variations", Arch. Rat. Mech. Anal., vol. 20, pp. 251-258, 1965.

2. KIUGE, R.

"Optimal Control with Minimum Problems and Varia tional Inequalities", Lecture Notes in Computer Science, Optim. Techn. IFIP Technical Conference, Springer, Berlino-Heidelberg-New York, 1975.

3. LIONS,J.I. "Optimal Control of Systems Governed by Partial Differential Equations", Springer, Berlino-Heidel berg, 1971.

4. IIONS,J.I. "Quelques méthodes de résolution des problèmes aux limites non linéaires", Dunod, Gauthier-Vil lers, 1969.

5. MOSCO,U. "Convergence of Convex Sets and of Solutions of Variational Inequalities", Adv, in Math., vol. 3, pp. 510-585, 1969 .

6. YVON,J.P. "Optimal Control of Systems Governed by Variatio nal Inequalities", Leoture Notes in Computer Science, 5 Conference on Optim. Techn., PertI, Springer, Berlin-Heidelberg-New York, 1973. 